\title{
Evaluation of Knowledge and Attitude Toward HPV and Vaccination Among Medical Staff, Medical Students, and Community Members in Fujian Province
}

This article was published in the following Dove Press journal:

Risk Management and Healthcare Policy

\author{
Chang Yu (ID) \\ Lihua Chen (D) ${ }^{\prime}$ \\ Guanyu Ruan (D) ${ }^{1,2}$ \\ Jian An (D) ${ }^{\prime}$ \\ Pengming Sun (D) ${ }^{1,2}$ \\ 'Laboratory of Gynecologic Oncology, \\ Fujian Provincial Maternity and Child \\ Health Hospital, Affiliated Hospital of \\ Fujian Medical University, Fuzhou, Fujian, \\ People's Republic of China; ${ }^{2}$ Key \\ Laboratory of Fujian Maternal and \\ Pediatric Major Disease Research, Fujian \\ Provincial Maternity and Child Health \\ Hospital, Affiliated Hospital of Fujian \\ Medical University, Fuzhou, Fujian, \\ People's Republic of China
}

Correspondence: Pengming Sun Key Laboratory of Fujian Maternal and Pediatric Major Disease Research, Fujian Provincial Maternity and Child Health Hospital, Affiliated Hospital of Fujian

Medical University, Fuzhou, Fujian,

People's Republic of China

Tel +86 59| 87558732

Fax+8659I8755 I247

Email sunfemy@hotmail.com
Purpose: To evaluate the level of human papilloma virus (HPV)-related knowledge and vaccination willingness of people in Fujian Province, and to explore the factors influencing doctors' recommendation of HPV vaccine.

Methods: We conducted two cross-sectional surveys in Fujian Province. The study cohort included 248 medical staff and medical students and 1001 community members. Descriptive statistics were used to identify the general demographics of the participants, along with their knowledge and attitudes regarding HPV and vaccination. Analyses were conducted to identify factors associated with willingness to vaccinate and willingness to recommend vaccination

Results: The level of HPV knowledge in Fujian province was found to be low, but more than $80 \%$ of participants would like to accept HPV vaccine. Medical staff had a higher willingness to recommend HPV vaccine than students (OR=4.696, CI: 2.698-8.175), which may be related to work experience and acceptance of vaccine price but not to knowledge level.

Conclusion: Knowledge of HPV in our community population appears to be lower than that in other regions in China. We suggest that a lack of knowledge may not be the main factor affecting the willingness to vaccinate, but we still needed to raise the level of knowledge about HPV to prevent misunderstandings. When the level of knowledge is high, education and per capita household income are not important factors influencing the willingness to vaccinate; only acceptance of vaccine prices significantly affects the willingness to vaccinate. Inclusion of the HPV vaccine in the national immunization program could help to alleviate public concerns regarding the vaccine to change present situation.

Keywords: perception, HPV, vaccine, knowledge, recommend

\section{Introduction}

Cervical cancer is the fourth most lethal human malignancy and the leading cause of death among females around the world; with especially high incidence in developing countries. ${ }^{1}$ There were roughly 569,847 new cases and 311,365 deaths associated with cervical cancer in 2018 alone. ${ }^{1}$ In mainland China, the rate of cervical cancer is estimated to be about 15.4 per 100,000 people, based on 2018 data, with the mortality rate being as high as 6.9 per $100,000 .{ }^{2}$ Persistent high-risk human papillomavirus (HR-HPV) infection is amajor cause of cervical cancer. ${ }^{3}$ 
According to the recommendations of the World Health Organization (WHO), HPV vaccines are effective prevention for cervical cancer and precancerous cervical lesions caused by carcinogenic HPV infection. ${ }^{4}$ Since 2017, three kinds of vaccine have been approved by China's Food and Drug Administration (FDA) for primary prevention of cervical cancer. ${ }^{2}$ We have estimated that only about $1 \%$ of eligible girls and women of appropriate age have been administered the HPV vaccine in mainland China as of the first half of 2019. ${ }^{5}$ However, in countries that include the HPV vaccine in their national immunization programs, such as Australia, the rate is as high as $86 \%{ }^{6}$ In addition to policy factors that influence vaccination rates, other barriers to vaccination include costs, vaccine availability, and lack of awareness. ${ }^{7}$ There are a few reports on the willingness to HPV vaccinate amongst Chinese community populations but do not include medical staff. ${ }^{8-10}$ The purpose of this study was to investigate the knowledge about HPV vaccines and willingness to accept or recommend HPV vaccination among medical staff, medical students, and community members in Fujian Province. Results from this study will help us to understand the factors influencing decisions and recommendations made by medical workers regarding vaccines and provide suggestions and evidence for health decision-makers.

\section{Methods}

\section{Study Design and Population Study Design}

Two cross-sectional surveys were conducted in 2016-2019 and compiled for summary analysis. Of the 68 counties in Fujian Province, 7 counties were selected randomly for a paper survey of community members with the assistance of the local Maternity and Children's Health Hospital. ${ }^{7}$ There were 1001 young women in the community who volunteered to participate in the study and these data were used for secondary analysis. ${ }^{7}$ The remainder of the data was across-sectional survey of medical staff and medical students in the Fujian Province of China conducted between April and July2019. For this study, medical staff was defined as individuals qualified to deliver healthcare services (ie, doctors, nurses, pathologists, pharmacists, radiologists, and laboratory physicians). A 41-question questionnaire was prepared based on previously published questionnaires. $^{7,8}$ The main content of the interviews included: (1) Characteristics; (2) Knowledge about HPV and HPV vaccination; (3) Reasons for willingness or unwillingness to be vaccinated and accepted cost; (4)
Factors associated with willingness to promote HPV vaccination, etc. The section investigating awareness of HPV consisted of six closed questions that could be answered "yes", "no", or "not clear" that assessed the respondent's knowledge of HPV. The frequencies and percentages of accurate responses were tabulated.

The online questionnaires were distributed through the mobile app WeChat. With sampling based on convenience, participants completed and submitted the questionnaire via mobile telephone. Each submitted questionnaire contained information, such as code, date, and time spent to complete the questionnaire, to ensure authenticity, validity, and completeness.

Random sampling among healthcare professionals was not feasible given the financial and time limitations of the study. The healthcare professionals included medical staff from various regions of Fujian Province and medical students from Fujian Medical University. Medical students were undergraduate in their 4-5 grade or postgraduate in their 13 grade (would graduate in 2020-2023). We also selected lectures to invite the healthcare professionals to complete the questionnaire anonymously. Obtain informed consent by written explanation and oral communication prior to data collection. Subjects were gender-neutral, participated voluntarily, and independently completed the questionnaire.

\section{Data Analysis}

A total of 1281 questionnaires were received, 5 participants were excluded from the study because their work did not conform to healthcare professionals inclusion criteria and 27 participants were excluded because of monitoring problems. The overall validity rate of participants was $97.5 \%$. The healthcare professionals received no financial incentives for their participation. Data were analyzed using SPSS version 19.0 (IBM Corp, Armonk, NewYork). The primary outcome measured in this study were HPV knowledge, intention to receive the HPV vaccine, and intention to recommend HPV vaccination. Descriptive statistics were used to identify the general demographics of the participants, along with their knowledge and attitudes regarding HPV and vaccination. To compare the medical staff, medical students, and community attitudes toward HPV vaccination, Pearson's correlations and Fisher's exact tests were used. A P value $<0.05$ was considered statistically significant. Calculate the chi-square and odds ratios of the factors affecting the willingness of medical personnel to recommend HPV vaccines. Ethical approval was obtained from the Fujian Provincial 
Maternity and Children's Hospital, an affiliated hospital of Fujian Medical University (approval number: 2016-019).

\section{Results}

\section{Participant Characteristics}

A summary of the participants' characteristics is provided in Table 1 (more complete demographic characteristics in sup plementary Table 1). Participants were divided into three groups: medical staff, medical students, and community members. The mean age of the community, medical student, and medical staff participants was $29.5 \pm 3.3,24.0 \pm 2.9$, and $38.4 \pm 8.4$ years, respectively. Nearly all of respondents were female $(96.4 \%)$ and $61.2 \%$ were citizens. The vast majority of healthcare professionals $(97.2 \%)$ had achieved at least junior college education, compared to only $45 \%$ of the community population who had a comparable level of education. The two data sets were largely mutually exclusive, as they were sourced from different populations. The distribution of family per capita monthly income in each group was different, with the majority of medical staff and the community earning $¥ 3001-5000$, whereas the per capita income of medical students was mostly below $¥ 3,000$ yuan.

\section{Knowledge of HPV and HPV Vaccination}

Details of the respondents' knowledge of HPV are reported in Table 2. Six items were thought to be related to HPV knowledge; univariate analysis found that the differences among the three groups were statistically significant. Among the community population, $44.9 \%$ expressed "Knows about HPV", but actually their knowledge was weak: $13.8 \%$ of them had just heard the name and only $30.7 \%$ could link HPV to sexually transmitted diseases. On questions regarding HPV-related knowledge, medical staff and students performed better than the community population $(\mathrm{P}<0.001)$. There was no difference in the performance of medical

Table I Demographic Characteristics of Participants

\begin{tabular}{|c|c|c|c|c|c|c|c|c|}
\hline Characteristics* & & $\begin{array}{l}\text { Medical } \\
\text { Staff }^{\mathrm{a}}(\mathrm{A}) \\
\mathrm{N}=\mid 46 \\
(\%)\end{array}$ & $\begin{array}{l}\text { Medical } \\
\text { Student (B) } \\
\mathrm{N}=102\end{array}$ & $\begin{array}{l}\text { Community } \\
\text { Population (C) } \\
\mathrm{N}=1001\end{array}$ & $\begin{array}{l}P(A, B \text {, } \\
\text { C) }\end{array}$ & $\begin{array}{l}\mathbf{P}(\mathbf{A}, \\
\mathbf{B})\end{array}$ & $\begin{array}{l}P(A, \\
\text { C) }\end{array}$ & $\begin{array}{l}P(B, \\
C)\end{array}$ \\
\hline Age range (years) & $\begin{array}{l}\leq 25 \\
26-30 \\
31-35 \\
36-40 \\
\geq 41\end{array}$ & $\begin{array}{l}5(3.4) \\
32(21.9) \\
22(15.1) \\
15(10.3) \\
72(49.3)\end{array}$ & $\begin{array}{l}87(85.3) \\
15(14.7) \\
0(0.0) \\
0(0.0) \\
0(0.0)\end{array}$ & $\begin{array}{l}122(12.2) \\
433(43.3) \\
446(44.6) \\
0(0.0) \\
0(0.0)\end{array}$ & $<0.001$ & $<0.001$ & $<0.001$ & $<0.001$ \\
\hline Gender & $\begin{array}{l}\text { Male } \\
\text { Female }\end{array}$ & $\begin{array}{l}15(10.3) \\
131(89.7)\end{array}$ & $\begin{array}{l}30(29.4) \\
72(70.6)\end{array}$ & $\begin{array}{l}0(0.0) \\
1001(100.0)\end{array}$ & $<0.001$ & $<0.001$ & $<0.001$ & $<0.001$ \\
\hline Education & $\begin{array}{l}\text { Master and above } \\
\text { Undergraduate/ } \\
\text { Junior college } \\
\text { High school/ } \\
\text { Technical } \\
\text { secondary school } \\
\text { Junior middle } \\
\text { school } \\
\text { Primary School } \\
\text { Illiterate }\end{array}$ & $\begin{array}{l}13(8.9) \\
127(87.0) \\
6(4.1) \\
0(0.0) \\
0(0.0) \\
0(0.0)\end{array}$ & $\begin{array}{l}42(4 I .2) \\
59(57.8) \\
I(I .0) \\
0(0.0) \\
0(0.0) \\
0(0.0)\end{array}$ & $\begin{array}{l}15(1.5) \\
435(43.5) \\
297(29.7) \\
227(22.7) \\
24(2.4) \\
3(0.3)\end{array}$ & $<0.001$ & $<0.001$ & $<0.001$ & $<0.001$ \\
\hline Birthplace & $\begin{array}{l}\text { Metropolitan } \\
\text { Rural area }\end{array}$ & $\begin{array}{l}I I I(76.0) \\
35(24.0)\end{array}$ & $\begin{array}{l}57(55.9) \\
45(44.1)\end{array}$ & $\begin{array}{l}596(59.5) \\
405(40.5)\end{array}$ & $<0.001$ & $<0.001$ & $<0.001$ & 0.526 \\
\hline $\begin{array}{l}\text { Average familyincome } \\
\text { b (per capita monthly) }\end{array}$ & $\begin{array}{l}<¥ 3000 \\
¥ 3001-5000 \\
¥ 5001-8000 \\
>¥ 8000\end{array}$ & $\begin{array}{l}16(11.0) \\
59(40.4) \\
38(26.0) \\
33(22.6)\end{array}$ & $\begin{array}{l}43(42.2) \\
28(27.5) \\
18(17.6) \\
13(12.7)\end{array}$ & $\begin{array}{l}223(22.3) \\
427(42.7) \\
203(20.3) \\
148(\mid 4.8)\end{array}$ & $<0.001$ & $<0.001$ & 0.002 & $<0.001$ \\
\hline
\end{tabular}

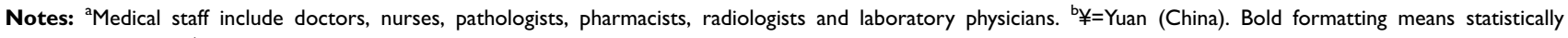
significant $(p<0.05)$. *More complete demographic characteristics in supplementary Table I. 
Table 2 Knowledge About HPV and HPV Vaccination Among the Participants Demographic

\begin{tabular}{|c|c|c|c|c|c|c|c|}
\hline $\begin{array}{l}\text { Knowledge Question (Correctly } \\
\text { Answered,\%) }\end{array}$ & $\begin{array}{l}\text { Medical } \\
\text { Staff }{ }^{a}(A) \\
N=145^{*}\end{array}$ & $\begin{array}{l}\text { Medical } \\
\text { Student (B) } \\
\mathrm{N}=98^{*}\end{array}$ & $\begin{array}{l}\text { Community } \\
\text { Population (C) } \\
\mathrm{N}=449 *\end{array}$ & $\begin{array}{l}P(A, B \text {, } \\
\text { C) }\end{array}$ & $\begin{array}{l}\mathbf{P}(\mathbf{A}, \\
\text { B) }\end{array}$ & $\begin{array}{l}P(A, \\
\text { C) }\end{array}$ & $\begin{array}{l}\mathbf{P}(\mathbf{B}, \\
\text { C) }\end{array}$ \\
\hline Knows about HPV* & $145(99.3)$ & $98(96.1)$ & 449(44.9) & $<0.001$ & $0.162^{b}$ & $<0.001$ & $<0.001$ \\
\hline $\begin{array}{l}\text { When HPV is mentioned, you will think of } \\
\text { (multi-choice): } \\
\text { Sexually transmitted diseases } \\
\text { Genital warts } \\
\text { Cervical cancer } \\
\text { HIV (wrong answer) } \\
\text { No knowledge about HPV(wrong answer) }\end{array}$ & $\begin{array}{l}65(44.8) \\
82(56.6) \\
138(95.2) \\
126(86.9) \\
145(100.0)\end{array}$ & $\begin{array}{l}59(60.2) \\
64(65.3) \\
96(98.0) \\
93(94.9) \\
98(100.0)\end{array}$ & $\begin{array}{l}138(30.7) \\
144(32.1) \\
346(77.1) \\
410(91.3) \\
449(86.9)\end{array}$ & $\begin{array}{l}<0.001 \\
<0.001 \\
<0.001 \\
0.093 \\
<0.001\end{array}$ & $\begin{array}{l}\mathbf{0 . 0 2 6} \\
0.184 \\
0.320^{b} \\
\mathbf{0 . 0 4 8} \\
0.309\end{array}$ & $\begin{array}{l}0.002 \\
<0.001 \\
<0.001 \\
0.146 \\
<0.001\end{array}$ & $\begin{array}{l}<0.001 \\
<0.001 \\
<0.001 \\
0.307 \\
<0.001\end{array}$ \\
\hline Knows that men can be infected by HPV & $|4|(97.2)$ & $90(91.8)$ & $225(50.1)$ & $<0.001$ & $0.072^{b}$ & $<0.001$ & $<0.001$ \\
\hline $\begin{array}{l}\text { How is the HPV transmitted? } \\
\text { Sexual contact } \\
\text { Indirect contact } \\
\text { Both (correct answer) } \\
\text { Not clear }\end{array}$ & $\begin{array}{l}53(36.6) \\
I(0.7) \\
91(62.8) \\
0(0.0)\end{array}$ & $\begin{array}{l}5 I(52.0) \\
0(0.0) \\
46(46.9) \\
I(1.0)\end{array}$ & $\begin{array}{l}185(41.2) \\
7(1.6) \\
175(39.0) \\
81(18.0)\end{array}$ & $<0.001$ & 0.018 & $<0.001$ & 0.173 \\
\hline $\begin{array}{l}\text { What is the prevalence of the HPV in the } \\
\text { population? } \\
\text { More than } 70 \% \\
50-70 \% \\
30-50 \% \\
\text { Less than } 30 \% \text { (correct answer) } \\
\text { Not clear }\end{array}$ & $\begin{array}{l}24(16.6) \\
41(28.3) \\
33(22.8) \\
34(23.4) \\
13(9.0)\end{array}$ & $\begin{array}{l}11(I 1.2) \\
22(22.4) \\
26(26.5) \\
17(17.3) \\
22(22.4)\end{array}$ & $\begin{array}{l}39(8.7) \\
68(15.1) \\
83(18.5) \\
73(16.3) \\
186(41.43)\end{array}$ & 0.141 & 0.266 & 0.062 & 0.880 \\
\hline $\begin{array}{l}\text { What are the risks of HPV infection? } \\
\text { High risk (correct answer) } \\
\text { Low risk } \\
\text { No risk } \\
\text { Not clear }\end{array}$ & $\begin{array}{l}105(72.4) \\
35(24.1) \\
0(0.0) \\
5(3.4)\end{array}$ & $\begin{array}{l}62(63.3) \\
27(27.6) \\
3(3.1) \\
6(6.1)\end{array}$ & $\begin{array}{l}345(77.0) \\
22(4.9) \\
I(0.2) \\
80(17.9)\end{array}$ & 0.019 & 0.158 & 0.316 & 0.007 \\
\hline
\end{tabular}

Notes: *Denotes the number of the participants who had heard of HPV would answered the below questions. Bold formatting means statistically significant ( $p<0.05$ ). ${ }^{a}$ Medical staff include doctors, nurses, pathologists, pharmacists, radiologists and laboratory physicians. ${ }^{b}$ More than $20 \%$ of cells in this subtable have expected cell counts less than 5. Chi-square results may be invalid.

staff and medical students, except for on the questions "How is the HPV transmitted?" on which medical staff performed better than students $(\mathrm{P}<0.05)$. We found that alot of medical staff and students involved in the study did not know that HPV could be transmitted through direct or indirect contact, and only $62.8 \%$ and $46.9 \%$, respectively, answered correctly. In addition, there is not enough knowledge on the issue of HPV infection rate (only $17.9 \%$ of people answered correctly, and most respondents overestimated the infection rate).

\section{Vaccination Willingness and Reasons}

In this survey, the intention to receive the HPV vaccine was highest among medical staff $(91.8 \%)$, followed by medical students $(90.2 \%)$ and the wider community $(83.6 \%)$. Interestingly, there was a significant difference in the willingness of medical staff and the community to vaccinate, but the difference between the community and medical students was not statistically significant. The vast majority (79.8\%) of the community population was willing to pay less than $¥ 500$ for the vaccine, while half of the medical staff would pay more than $¥ 500$. The acceptance of vaccine prices by medical students was between the other two groups, with nearly $60 \%$ expecting the vaccine price to be less than $¥ 500$. In addition, about $10 \%$ of medical staff or medical students refused to receive the HPV vaccine, compared to $16.3 \%$ in the community. 
The reasons determining the willingness to vaccinate were different between the three groups, with medical staff selecting "Doubts on the source" and "Vaccination causes risks" most frequently, medical students choosing "Vaccines are too expensive" most frequently, and "Not yet widely used" selected most often by the community members. The majority $(85.2 \%)$ of respondents expressed their willingness to be vaccinated, and almost $81.5 \%$ of the respondents said they believed HPV vaccination would be beneficial. Other common reasons given were "Worried about HPV infection" and "Worried about cervical cancer". Relatively few people indicated "Worry about genital warts" as a reason for vaccination.

\section{Willingness of the Medical Staff and Medical Student to Recommend HPV Vaccination}

For this analysis, we put healthcare professionals who were neutral, opposed, or unclear about the recommended vaccine in the same category- will not actively recommend the vaccine. In our survey, $63.7 \%$ of medical staff indicated their willingness to recommend vaccines, and the variables that influenced the decision to recommend HPV vaccination are shown in Table 3.

We first screened the influencing factors with aone-way ANOVA and calculated their odds ratio (OR). There was asignificant correlation between the willingness to vaccinate with HPV vaccine and the recommended willingness of HPV vaccine ( $\mathrm{p}=0.002$, OR: 4.314 (1.687-11.032)). The willingness of women to recommend HPV vaccination was twice as high as that of men. Medical staff were more willing to recommend vaccines than medical students [OR: 4.696 (2.698-8.175)]. With the increase in professional titles, the willingness of medical staff to recommend HPV gradually increased; deputy chief physicians were 11 times more likely than interns (medical students) to recommend vaccination. Although the number of chief physicians in the cohort was small, the proportion of those willing to recommend vaccination was as high as $100 \%$. Similar results also appear in terms of age. People

Table 3 Reasons for Willing and Unwilling to Be Vaccinated and Accepted Cost

\begin{tabular}{|c|c|c|c|c|c|c|c|}
\hline Item & $\begin{array}{l}\text { Medical } \\
\text { Staff }^{a} \\
\text { (A) } \\
N=146\end{array}$ & $\begin{array}{l}\text { Medical } \\
\text { Student } \\
\text { (B) } \\
N=102\end{array}$ & $\begin{array}{l}\text { Community } \\
\text { Population } \\
\text { (C) } \mathrm{N}=100 \mathrm{I}\end{array}$ & $P(A, B, C)$ & $\mathbf{P}(\mathbf{A}, \mathrm{B})$ & $P(A, C)$ & $P(B, C)$ \\
\hline Willing to take the HPV vaccine & |34(9|.8) & $92(90.2)$ & $838(83.7)$ & 0.012 & 0.821 & 0.013 & 0.114 \\
\hline $\begin{array}{l}\text { Reasons for unwilling to be vaccinated } \\
\text { I do not have risk } \\
\text { Do not think vaccination works } \\
\text { It has not been widely used } \\
\text { Vaccination causes risks } \\
\text { Doubts on the source } \\
\text { The vaccine is expensive } \\
\text { The process of vaccination is cumbersome }\end{array}$ & $\begin{array}{l}12(8.2) \\
4 / 12 \\
1 / 12 \\
5 / 12 \\
6 / 12 \\
6 / 12 \\
4 / 12 \\
3 / 12\end{array}$ & $\begin{array}{l}10(9.8) \\
4 / 10 \\
2 / 10 \\
2 / 10 \\
1 / 10 \\
0 / 10 \\
8 / 10 \\
2 / 10\end{array}$ & $\begin{array}{l}163(16.3) \\
51 / 163 \\
19 / 163 \\
112 / 163 \\
58 / 163 \\
27 / 163 \\
1 \\
1\end{array}$ & $<0.001^{b}$ & $0.073^{b, c}$ & $<0.001^{b, c}$ & $<0.00 \mathrm{I}^{\mathrm{b}, \mathrm{c}}$ \\
\hline $\begin{array}{l}\text { Reasons for willing to be vaccinated } \\
\text { Self benefit } \\
\text { Fear of infecting HPV } \\
\text { Fear of cervical cancer in yourself/your } \\
\text { partner } \\
\text { Fear of having genital warts }\end{array}$ & $\begin{array}{l}134(91.8) \\
118 / 134 \\
69 / 134 \\
70 / 134 \\
40 / 134\end{array}$ & $\begin{array}{l}92(90.2) \\
78 / 92 \\
53 / 92 \\
40 / 92 \\
26 / 92\end{array}$ & $\begin{array}{l}838(83.7) \\
671 / 838 \\
391 / 838 \\
393 / 838 \\
222 / 838\end{array}$ & $<0.001$ & $0.076^{c}$ & $<0.00 I^{c}$ & $0.004^{c}$ \\
\hline $\begin{array}{l}\text { Accepted costs (3 does) } \\
\quad<¥ 200 \\
¥ 200-500 \\
¥ 500-1000 \\
>¥ 1000\end{array}$ & $\begin{array}{l}146 \\
(100.0) \\
26 / 146 \\
46 / 146 \\
41 / 146 \\
33 / 146\end{array}$ & $\begin{array}{l}102(100.0) \\
21 / 102 \\
38 / 102 \\
30 / 102 \\
13 / 102\end{array}$ & $\begin{array}{l}1001(100.0) \\
416 / 1001 \\
382 / 1001 \\
126 / 1001 \\
77 / 1001\end{array}$ & $<0.001$ & 0.264 & $<0.001$ & $<0.001$ \\
\hline
\end{tabular}

Notes: ${ }^{a}$ Medical staff include doctors, nurses, pathologists, pharmacists, radiologists and laboratory physicians. ${ }^{b}$ More than $20 \%$ of cells in this subtable have expected cell counts less than 5 . Chi-square results may be invalid. 'Likelihood ratio for aChi-square test. Bold formatting means statistically significant ( $\mathrm{P}<0.05$ ). 
with different recommendation intentions had different average family incomes, but increased income was not linearly related to the willingness to recommend vaccination. Interestingly, there was no correlation between education and willingness to recommend vaccination.

\section{Discussion}

In the current study, we found that knowledge of HPV is limited in the community at large. Less than half of the community population had heard of HPV, and more than $10 \%$ of them said that they had no more knowledge except to know the name. Knowledge of HPV in our community population appeared similarly to other regions in China, and far below what has been reported in other countries,8,11-13 which could be improved by media outreach. Knowledge of HPV infection and transmission was worse among medical students compared to medical staff. It cannot be ignored that about half of medical staff and students ignored or were unaware that HPV can be transmitted via indirect contact. In New Zealand, only $11 \%$ of health practitioners either thought or were not sure whether HPV can be passed by genital skin-to-skin contact. ${ }^{14}$ This is a stark reminder of the lack of education regarding HPV in China, and it is urgent to improve the knowledge level of the community. Prior to this, the HPV knowledge that medical staff lacks should be targeted to improve. A Swedish study suggested that $68.3 \%$ of patients prefer to get preventive knowledge about HPV from doctors, which may be a method that helps to increase awareness in China. ${ }^{3}$ More education is needed to ensure that health professionals and the public do not inadvertently spread misinformation. ${ }^{15}$

Data from amulticenter study of the Chinese population showed that both HPV type 16 and 18 vaccines prevented up to $84.5 \%$ of cervical cancer and $72 \%$ of high-grade cervical intraepithelial neoplasia. Because the HPV vaccine has not received financial support from the Chinese government, vaccination depends on the willingness of age-appropriate girls and women to accept the vaccine. ${ }^{16}$ In our survey, we found a significant difference in the level of knowledge of medical staff and community members. But there was no significant difference in the willingness of community members and medical students to vaccinate, both groups were less willing to vaccinate than medical staff. This suggests that a lack of knowledge in younger populations (Table 4) may not be the main factor affecting the willingness to vaccinate. Because we cannot rule out the impact of age on the willingness of medical staff and community members to accept HPV vaccination, the importance of knowledge of HPV cannot be accurately estimated. To further explore the factors affecting the willingness to vaccinate, we selected doctors and medical students with no significant differences in knowledge background. As there was no difference in the willingness of these groups to vaccinate, we considered the two groups as a single population and compared that combined population with factors that also exist in the community (Table 5). From our results, we can conclude that when the level of knowledge is high, education level and household income are not important factors affecting the willingness to vaccinate. Only the price of the vaccine had a significant effect on willingness to vaccinate, which is consistent other surveys. ${ }^{17}$

Studies have shown that physician recommendation is an important and consistent predictor of vaccine uptake. ${ }^{18}$ Astudy in New Zealand reported that $96.5 \%$ of health workers agreed or strongly agreed that they would recommend the HPV vaccine, compared to just $63 \%$ in our study. ${ }^{14}$ Further analysis (Table 4) found that medical staff had ahigher willingness to recommend vaccination than medical students and that likelihood to recommend vaccination was linearly correlated to both job title and age. Increases in age and title are typically associated with a greater degree of clinical knowledge and experience. Those medical staff who are willing to get vaccinated have a higher tendency to recommend the vaccine. For healthcare professional, women were more willing to recommend vaccination than men. Medical staff accepted a higher price for the vaccine price than community members, most likely because of their medical background. However, the majority of people indicated an acceptable budget for the vaccine that is lower than the lowest-priced vaccine in the Chinese market (Cervarix, GlaxoSmithKline Biologicals SA, Rixensart, Belgium; $¥ 1827 / 3$ doses).

There are inherent limitations associated with the use of questionnaires with self-reported information, so conclusions and claims based on self-reported data may contain bias. Social expectations may affect the sample. Even if the questionnaire is anonymous and the project is carefully designed, participants may answer questions in asocially desirable manner. Second, almost all the participants in our study were women. Women who are willing to receive the HPV vaccine are more likely to respond to the survey than women who are not willing to receive the HPV vaccine. This factor will lead to overestimation of HPVrelated knowledge and vaccination willingness. The analysis can only provide statistically relevant evidence between explanatory variables and questions of common interest. Although online surveys have alower response rate than paper survey forms, they are more cost-effective and have fewer missing values. ${ }^{19}$ Fourth, the questionnaire for community populations was collected in 
Table 4 Factors Associated with Willing to Promote HPV Vaccination Among the Medical Staff and Medical Student

\begin{tabular}{|c|c|c|c|c|c|c|c|}
\hline \multirow[t]{2}{*}{ Variables } & \multicolumn{4}{|c|}{ Willing to Promote $(\mathrm{N}=\mathbf{2 4 8})$} & \multirow[t]{2}{*}{ Chi-Square } & \multirow[t]{2}{*}{$\mathbf{P}$} & \multirow[t]{2}{*}{ Code OR } \\
\hline & $n_{\text {yes }}=158$ & $\%$ & $n_{n o}=90$ & $\%$ & & & \\
\hline Age range (years) & & & & & 36.516 & $<0.001^{\mathrm{a}}$ & \\
\hline$\leq 25$ & 42 & 45.7 & 50 & 54.3 & & & I \\
\hline $26-30$ & 26 & 55.3 & 21 & 44.7 & & & $1.474(0.727-2.987)$ \\
\hline $31-35$ & 14 & 63.6 & 8 & 36.4 & & & $2.083(0.797-5.445)$ \\
\hline $36-40$ & II & 73.3 & 4 & 26.7 & & & $3.274(0.97|-| I .04 I)$ \\
\hline$\geq 41$ & 65 & 90.3 & 7 & 9.7 & & & I I.054(4.58I-26.678) \\
\hline Gender & & & & & 4.469 & 0.035 & \\
\hline Male & 22 & 48.9 & 23 & 51.1 & & & 1 \\
\hline Female & 136 & 67.0 & 67 & 33.0 & & & $2.122(1.104-4.080)$ \\
\hline Education & & & & & 2.228 & 0.518 & \\
\hline Master and above & 32 & 58.2 & 23 & 41.8 & & & - \\
\hline Undergraduate & 91 & 63.6 & 52 & 36.4 & & & - \\
\hline Junior college & 29 & 67.4 & 14 & 32.6 & & & - \\
\hline Technical secondary school & 6 & 85.7 & 1 & 14.3 & & & - \\
\hline Profession & & & & & 31.716 & $<0.001$ & \\
\hline Medical student & 44 & 43.1 & 58 & 56.9 & & & I \\
\hline Medical staff & 114 & 78.1 & 32 & 21.9 & & & $4.696(2.698-8.175)$ \\
\hline Average family income & & & & & 9.485 & 0.023 & \\
\hline$<¥ 3000$ & 30 & 50.8 & 29 & 49.2 & & & 1 \\
\hline$¥ 300 \mathrm{I}-5000$ & 62 & 71.3 & 25 & 28.7 & & & $2.397(1.202-4.780)$ \\
\hline$¥ 500 I-8000$ & 32 & 57.1 & 24 & 42.9 & & & $1.289(0.618-2.689)$ \\
\hline$>¥ 8000$ & 34 & 73.9 & 12 & 26.1 & & & $2.739(1.191-6.299)$ \\
\hline Willing to receive HPV vaccination & & & & & 10.620 & 0.002 & \\
\hline No & 7 & 31.8 & 15 & 68.2 & & & 1 \\
\hline Yes & 151 & 66.8 & 75 & 33.2 & & & $4.3 \mid 4(1.687-\mid I .032)$ \\
\hline Professional Title & & & & & 36.620 & $<0.00 I^{\mathrm{a}}$ & \\
\hline No technical titles (Intern physician) & 47 & 44.8 & 58 & 55.2 & & & I \\
\hline Resident & 31 & 62.0 & 19 & 38.0 & & & $2.013(1.011-4.008)$ \\
\hline Attending physician & 45 & 81.8 & 10 & 18.2 & & & $5.553(2.53|-| 2.185)$ \\
\hline Associate chief physician & 27 & 90.0 & 3 & 10.0 & & & II.106(3.172-38.893) \\
\hline Chief physician & 8 & 100.0 & 0 & 0.0 & & & - \\
\hline
\end{tabular}

Notes: $\mathrm{n}_{0}$ : Combine people with neutral, ambiguous and negative views into people who do not actively recommend HPV vaccine. Bold formatting means statistically significant. ${ }^{a}$ Linear by linear association. ${ }^{b}$ Continuity correction. Adash indicates that data are not available.

2016-2017, and knowledge and willingness to vaccinate may have changed in the study population.

This study also has important advantages. This study shows the difference of knowledge about HPV vaccine between community residents and medical staff, and the willingness of medical staff to recommend HPV vaccine. The survey methods used in our study have been adjusted for potential confounding factor and were suitable for specific demographic characteristics. The conclusions may help to improve vaccine coverage to reduce the incidence and death rate of HPV-related diseases.

\section{Conclusion}

In summary, we suggest that a lack of knowledge may not be the main factor affecting the willingness to vaccinate, but there is still ademonstrable lack of awareness of HPV among both healthcare professionals and the wider community. Therefore, efforts are needed to raise the level of knowledge about HPV through general education and to increase awareness to prevent misunderstandings. Our research suggests that when the level of knowledge is high, education and per capita household income are not important factors influencing the 
Table 5 Factors Affecting the Medical Staff and Medical Student Accepting or Rejecting HPV Vaccine

\begin{tabular}{|c|c|c|c|c|c|c|c|}
\hline \multirow[t]{2}{*}{ Item } & \multirow[t]{2}{*}{$\mathrm{N}=\mathbf{2 4 8}$} & \multicolumn{4}{|c|}{ Do You Want to Be Vaccinated Against HPV? } & \multirow[t]{2}{*}{ Chi-Square } & \multirow[t]{2}{*}{$\mathbf{P}$} \\
\hline & & $n_{\text {yes }}=226$ & $\%$ & $n_{n o}=22$ & $\%$ & & \\
\hline Accepted costs ( 3 does) & & & & & & 10.679 & 0.020 \\
\hline$<¥ 200$ (<US\$29.0) & 47 & 40 & 17.7 & 7 & 31.8 & & \\
\hline$¥ 200-500$ (US\$29.0-72.4) & 84 & 75 & 33.2 & 9 & 40.9 & & \\
\hline$¥ 500-1000($ US $\$ 72.4-144.9)$ & 71 & 65 & 28.8 & 6 & 27.3 & & \\
\hline$>¥ 1000$ (>US\$144.9) & 46 & 46 & 20.4 & 0 & 0.0 & & \\
\hline Expectations after vaccination with HPV & & & & & & 0.894 & 0.859 \\
\hline Not in the future & 22 & 19 & 9.4 & 3 & 13.6 & & \\
\hline Not for years & 62 & 56 & 24.8 & 6 & 27.3 & & \\
\hline Still possible & 155 & 143 & 63.3 & 12 & 54.5 & & \\
\hline Do not know & 9 & 8 & 3.5 & 1 & 4.5 & & \\
\hline What are the risks of HPV infection? & & & & & & 6.802 & 0.63 \\
\hline High risk & 168 & 158 & 69.9 & 10 & 45.5 & & \\
\hline Low risk & 62 & 52 & 23.0 & 10 & 45.5 & & \\
\hline No risk & 3 & 2 & 0.9 & 1 & 4.5 & & \\
\hline Not clear & 15 & 14 & 6.2 & 1 & 4.5 & & \\
\hline Education & & & & & & 1.717 & 0.721 \\
\hline Master and above & 55 & 50 & 22.1 & 0 & 0.0 & & \\
\hline Undergraduate & 143 & 131 & 58.0 & 5 & 22.7 & & \\
\hline Junior college & 43 & 38 & 16.8 & 12 & 54.5 & & \\
\hline Technical secondary school & 7 & 7 & 3.1 & 5 & 22.7 & & \\
\hline Average family income & & & & & & 2.740 & 0.445 \\
\hline$<¥ 3000$ & 59 & 51 & 22.6 & 8 & 36.4 & & \\
\hline$¥ 300 \mathrm{I}-5000$ & 87 & 79 & 35.0 & 8 & 36.4 & & \\
\hline$¥ 500 I-8000$ & 56 & 53 & 23.5 & 3 & 13.6 & & \\
\hline$>¥ 8000$ & 46 & 43 & 19.0 & 3 & 13.6 & & \\
\hline
\end{tabular}

Note: Bold formatting means statistically significant.

willingness to vaccinate; only acceptance of vaccine prices significantly affects the willingness to vaccinate. China faces the huge challenge to effectively prevent and control cervical cancer in a short time. Improvements in vaccine coverage require government leadership. Inclusion of the HPV vaccine in the national immunization program could help to alleviate public concerns regarding the vaccine. Furthermore, the cost of the vaccine must be reduced, which is in line with national interests and personal expectations. ${ }^{20,21}$

\section{Acknowledgments}

The authors thank the Maternity and Children's Health Hospital of Xianyou, Maternity and Children's Health Hospital of Shaowu, Maternity and Children's Health Hospital of Nanping, Maternity and Children's Health Hospital of Shishi, Maternity and Children's Health
Hospital of Jinjiang, and Maternity and Children's Health Hospital of Fuqing for helping with data collection.

\section{Disclosure}

The authors report no conflicts of interest in this work.

\section{References}

1. BrayF, FerlayJ , SoerjomataramI, SiegelRL, TorreLA, JemalA . Global cancer statistics 2018: GLOBOCAN estimates of incidence and mortality worldwide for 36 cancers in 185 countries. CA Cancer JClin . 2018;68(6):394-424. doi:10.3322/caac.21492

2. PanXF, LiR, PanA, LarsonH. Human papillomavirus vaccine approval in China: amajor step forward but challenges ahead . Lancet Infect Dis. 2016;16(12):1322-1323. doi:10.1016/S1473-3099(16)30450-9

3. Ahrlund-Richter A, ChengL, HuYOO, et al. Changes in cervical human papillomavirus (HPV) prevalence at ayouth clinic in stockholm, sweden, adecade after the introduction of the HPV vaccine. Front Cell Infect Microbiol. 2019;9:59. doi:10.3389/fcimb.2019.00059

4. Organization WH. Comprehensive cervical cancer prevention and control: ahealthier future for girls and women . [J]. Geneva Switzerland Who, 2013;28(3):271-272. 
5. Summary (publication) form for the batch of products issued by biological products; 2019. Available from: https://bio.nifdc.org.cn/ pqf/search.do?formAction=pqfGs. Accessed December 09, 2019.

6.HullB , HendryA , DeyA , BeardF , BrothertonJ , McIntyreP . Immunisation coverage annual report, 2015. Commun Dis Intell. 2018;2019(43).

7. ChenL, SongY, RuanG, etal. Knowledge and attitudes regarding HPV and vaccination among chinese women aged 20 to 35 years in fujian province: a cross-sectional study. Cancer Control. 2018;25 (1):1073274818775356. doi:10.1177/1073274818775356

8. LiJ , LiLK, MaJF , etal. Knowledge and attitudes about human papillomavirus (HPV) and HPV vaccines among women living in metropolitan and rural regions of China. Vaccine. 2009;27(8):1210 1215. doi:10.1016/j.vaccine.2008.12.020

9. BalochZ, YasmeenN , LiY, etal. Knowledge and awareness of cervical cancer, human papillomavirus (HPV), and HPV vaccine among HPV-infected chinese women. Med Sci Monit. 2017;23:4269-4277. doi:10.12659/MSM.903370

10. HanYF, ZhuangYN, LiY, FangY . Analysis of mothers' acceptance of HPV vaccination of adolescent girls in Xiamen. Zhonghua Yu fang Yi Xue Za Zhi [Chin JPrev Med] . 2018;52(1):38-42. doi:10.3760/ cma.j.issn.0253-9624.2018.01.008

11. AbuliziG, AbulimitiT, LiH, etal. Knowledge of cervical cancer and pap smear among Uyghur women from Xinjiang, China. BMC Women's Health. 2018;18(1):21. doi:10.1186/s12905-018-0512-5

12. TusiminM, YeeCL, RazakN, ZainolMI, MinhatHS, RejaliZ . Sociodemographic determinants of knowledge and attitude in the primary prevention of cervical cancer among University Tunku Abdul Rahman (UTAR) students in Malaysia: preliminary study of HPV vaccination. BMC Public Health. 2019;19(1):1454. doi:10.1186/s12889-019-7764-3

13. JainN, EulerGL, SheferA, LuP, YankeyD, MarkowitzL . Human papillomavirus (HPV) awareness and vaccination initiation among women in the United States, National Immunization Survey-Adult 2007. Prev Med. 2009;48(5):426-431. doi:10.1016/j.ypmed.2008. 11.010
14. ShermanSM , BartholomewK, DenisonHJ, etal. Knowledge, attitudes and awareness of the human papillomavirus among health professionals in New Zealand. PLoS One. 2018;13(12):e0197648. doi:10.1371/journal.pone.0197648

15. PatelH , Austin-Smith K, ShermanSM , TincelloD, MossEL . Knowledge, attitudes and awareness of the human papillomavirus amongst primary care practice nurses: an evaluation of current training in England. JPublic Health . 2017;39(3):601-608. doi:10.1093/ pubmed/fdw063

16. ChenW, ZhangX, MolijnA, etal. Human papillomavirus typedistribution in cervical cancer in China: the importance of HPV 16 and 18. Cancer Causes Control. 2009;20(9):1705-1713. doi:10.1007/ s10552-009-9422-z

17. TranBX, ThanPTQ, DoanTTN, etal. Knowledge, attitude, and practice on and willingness to pay for human papillomavirus vaccine: a cross-sectional study in Hanoi, Vietnam. Patient Prefer Adherence. 2018;12:945-954. doi:10.2147/PPA.S165357

18. VadaparampilST, KahnJA, SalmonD, etal. Missed clinical opportunities: provider recommendations for HPV vaccination for 1112year old girls are limited . Vaccine. 2011;29(47):8634-8641. doi:10.1016/j.vaccine.2011.09.006

19. EbertJF , HuibersL , ChristensenB , ChristensenMB . Paper- or webbased questionnaire invitations as amethod for data collection: crosssectional comparative study of differences in response rate, completeness of data, and financial cost . JMed Internet Res . 2018;20(1): e24. doi:10.2196/jmir.8353

20. LevinCE, SharmaM, OlsonZ, etal. An extended cost-effectiveness analysis of publicly financed HPV vaccination to prevent cervical cancer in China. Vaccine. 2015;33(24):2830-2841. doi:10.1016/j. vaccine.2015.02.052

21. WongCKH , ManKKC , IpP , KwanM , McGheeSM . Mothers' preferences and willingness to pay for human papillomavirus vaccination for their daughters: adiscrete choice experiment in HongKong. Value Health. 2018;21(5):622-629. doi:10.1016/j.jval.2017.10.012
Risk Management and Healthcare Policy

\section{Publish your work in this journal}

Risk Management and Healthcare Policy is an international, peerreviewed, open access journal focusing on all aspects of public health, policy, and preventative measures to promote good health and improve morbidity and mortality in the population. The journal welcomes submitted papers covering original research, basic science, clinical \& epidemiological studies, reviews and evaluations, guidelines, expert opinion and commentary, case reports and extended reports. The manuscript management system is completely online and includes a very quick and fair peer-review system, which is all easy to use. Visit http://www.dovepress.com/testimonials.php to read real quotes from published authors. 